\title{
Protée
}

\section{Voyage to Italy de Victor Burgin (fragments photographiques)}

\section{André Habib}

Volume 35, numéro 2, automne 2007

Imaginaire des ruines

URI : https://id.erudit.org/iderudit/017467ar

DOI : https://doi.org/10.7202/017467ar

Aller au sommaire du numéro

Éditeur(s)

Département des arts et lettres - Université du Québec à Chicoutimi

ISSN

0300-3523 (imprimé)

1708-2307 (numérique)

Découvrir la revue

Citer cet article

Habib, A. (2007). Voyage to Italy de Victor Burgin (fragments photographiques). Protée, 35(2), 55-64. https://doi.org/10.7202/017467ar d'utilisation que vous pouvez consulter en ligne.

https://apropos.erudit.org/fr/usagers/politique-dutilisation/ 


\title{
VOYAGE TO ITALY DE VICTOR BURGIN (fragments photographiques)
}

\author{
Une présentation d'André Habib
}

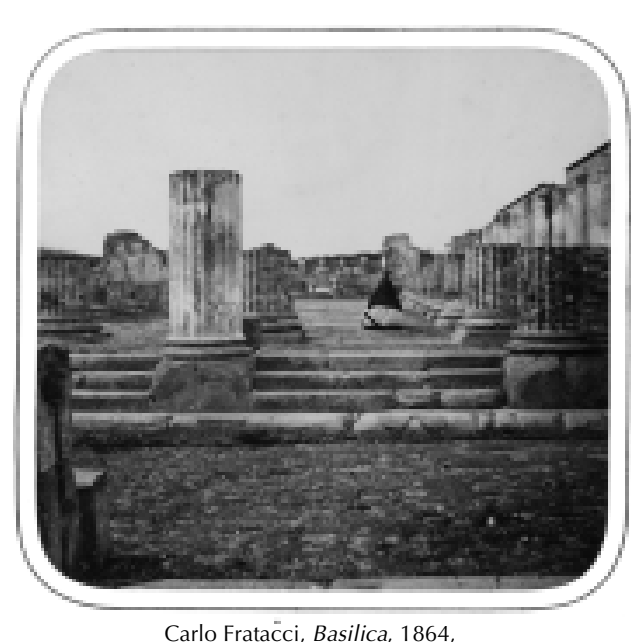

épreuve argentique à l'albumine, 17,4 × 18,1 cm*.

Voyage to Italy est une installation vidéo qu'il serait difficile de séparer des œuvres et des réflexions que, depuis trente ans, Burgin élabore sur la photographie et l'image animée, tout particulièrement dans ses essais récents In/Different Spaces ou The Remembered Film. Elle est également à comprendre comme un prolongement ou une ramification possible de ses installations, telles Elective Affinities (2000-2001), The Little House (2005) et Nietzsche's Paris (1999-2000) ou encore Listen to Britain (2001). Cette dernière vidéo, réalisée au lendemain du 11 septembre 2001, télescopait des vues « actuelles» du paysage de Kent avec un plan de A Canterbury Tale (1944) de Pressburger et Powell, tourné dans les même lieux. Le titre de l'œuvre faisait également écho au documentaire de Jennings, Listen to Britain (1941), réalisé durant les bombardements allemands. Chaque fois, il s'agit pour Burgin de déplier, de déployer un lieu à partir de sa mémoire: mémoire du lieu, mais aussi mémoire de l'artiste lui-même, qui s'imprègne du lieu, se laisse «impressionner », se livrant au jeu des associations libres, mêlant mémoire publique et mémoire privée. Listen to Britain fait jaillir, en creux, sans en livrer une image directe, la mémoire des décombres de la Seconde Guerre mondiale, à un moment de I'histoire collective hanté par l'image des tours jumelles effondrées. Bien qu'inscrit dans un autre temps et un autre lieu, Voyage to Italy approfondit à son tour cet imaginaire des ruines, de l'histoire et de la mémoire (culturelles et personnelles), tout en l'intégrant à une réflexion profonde sur l'ontologie de l'image photographique et cinématographique.

Présenté à Cologne à l'automne 2006, puis au Centre canadien d'architecture de Montréal à I'hiver 2007, Voyage to Italy trouve son origine dans une commande passée à divers artistes afin qu'ils réalisent une œuvre à partir d'une pièce ou d'un document des archives du CCA. Burgin choisit une photographie contenue dans un recueil de vues réalisées par Carlo Fratacci à Pompéi, en 1864. La photo en question, simplement intitulée Basilica, montre une basilique pompéienne dont ne restent que les marches et des colonnes en ruine. Au centre de l'image, précisément au point de fuite, se trouve une femme, portant une longue robe recouvrant une jupe en crinoline blanche et un chapeau en dentelle, de profil, quelque peu miniaturisée, de toute évidence «posée» là pour donner une idée de l'échelle de la construction effondrée. Plutôt que d'opter pour une explication «raisonnable» de la présence de la femme que la légende omet, Burgin est littéralement hanté par une autre explication, déraisonnable, délirante - comme celle de Norbert Hanold ${ }^{1}$, le personnage

\footnotetext{
* Planche non numérotée d'un album intitulé Principales Vues de Pompeii par Charles Fratacci, Naples 1864 comprenant 26 photographies. Collection Centre Canadien d'Architecture/Canadian Centre for Architecture, Montréal.
} 
de Gradiva de Wilhelm Jensen rendue célèbre par l'analyse de Freud de 1907: "cette femme est un "fantôme de midi" (mid-day ghost), elle n'est pas mentionnée parce qu'elle n'a pas été vue» (Burgin, 2006: 79; je traduis).

Il se rend donc à Pompéi, sur les traces de Fratacci, sur les traces de Hanold, sur les traces de Rossellini aussi qui, en 1953, allait tourner à Naples et à Pompéi un film décisif sur un couple qui s'étiole, un film hanté par les spectres du passé et de l'Histoire: Viaggio in Italia.

Une fois à Pompéi, littéralement guidé par l'image de Fratacci, Burgin réalise deux séries de photographies numériques, traçant un arc de $360^{\circ}$ à partir de deux points de vue: celui de la femme et celui du photographe. Ces deux séries d'images seront ensuite animées par ordinateur en un mouvement panoramique continu, pour constituer la trame principale de la vidéo. Il photographiera également, suivant un cadrage rigoureux et systématique, chacune des 24 colonnes corinthiennes qui encadraient la nef de la basilique, et disposera les photos tout autour de la salle de l'exposition, suivant un modèle qui rappelle les relevés architecturaux de la Renaissance. Toutes travaillées en noir et blanc à l'ordinateur, ces images produisent une impression mixte d'ancienneté et de nouveauté. L'immense espace vide de la basilique, cette série de colonnes tronquées, le ciel à la fois fixe et animé par le mouvement artificiel, mêlé à la précision terrifiante et quelque peu « atemporelle» du numérique, engendrent une véritable «spectralisation» du lieu de l'image. Et Pompéi - à la fois ville en ruine et ville intacte, lieu de vie, mais vie pétrifiée comme ces corps de plâtre noués dans l'étreinte que l'on y a retrouvée - est une ville propice à l'évocation des spectres. Une longue tradition artistique est là pour en témoigner, de Gautier à Bulwer-Lytton, de Jensen à Rossellini.

Voyage to Italy, 2006 (fragments photographiques).

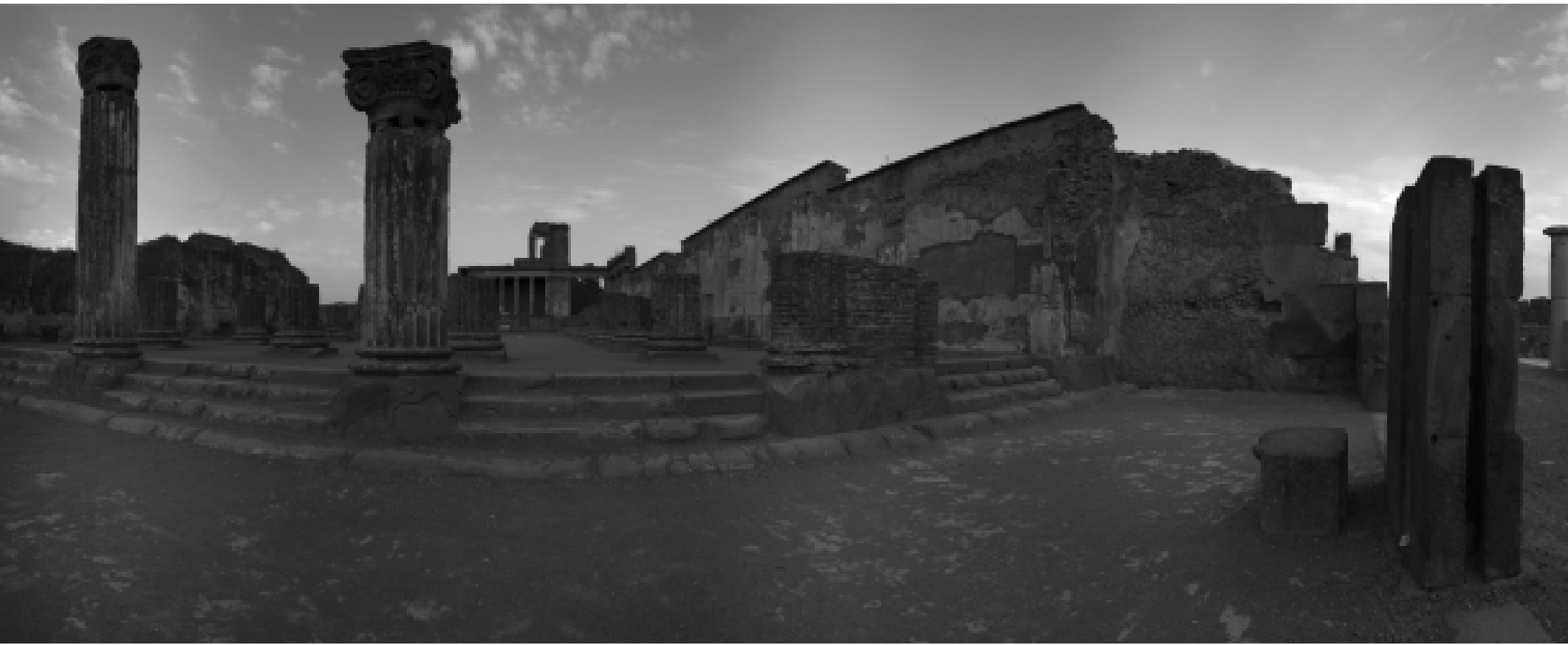


innombrables relations de voyage en Italie ... ou encore le montage photo-textuel que Burgin réalisa lui-même en 1982, Gradiva, conçu et élaboré à partir de la nouvelle de Jensen ${ }^{2}$.

C'est cette constellation d'associations qui constitue le Voyage de Burgin et qu'il nous invite à parcourir, à partir de notre propre travail de mémoire et d'imagination. Que peuvent un lieu, un paysage, une photographie d'un lieu? De quelle mémoire sont-ils habités, ou encore comment viennent-ils hanter notre mémoire, la mettre en mouvement, la faire travailler? Le Voyage to Italy de Burgin est le prolongement de cette interrogation qui concerne, au plus près, l'imaginaire de la ruine, d'hier et d'aujourd'hui.

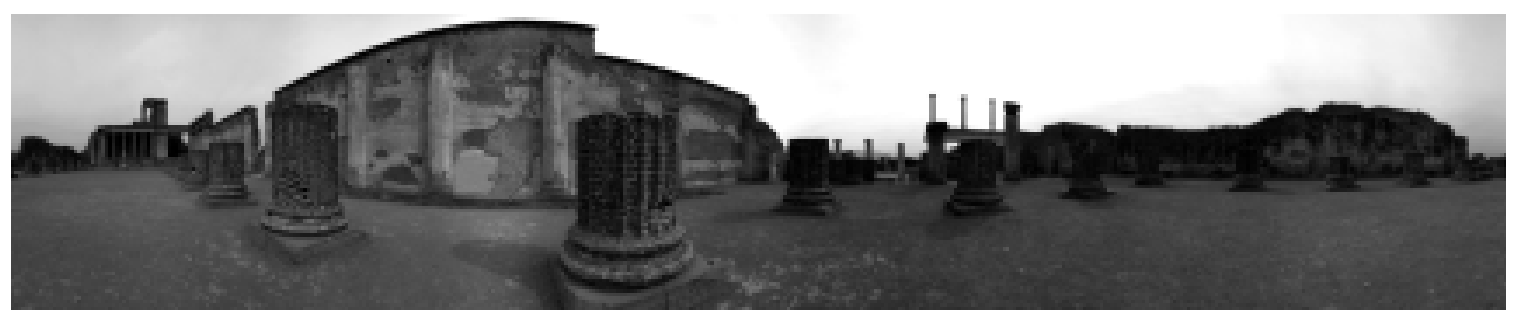

\section{NOTES}

1. Hanold, on se le rappellera, est un archéologue obsédé par un bas-relief montrant une jeune femme marchant, le pied arrière légèrement arqué. Incapable de trouver une même grâce dans la démarche des filles dans la ville où il se trouve, et après avoir fait un rêve où il assiste à l'éruption du volcan et à la mort de celle qu'il nomme Gradiva, il décide finalement de se rendre à Naples puis à Pompéi pour retrouver sa trace, une trace de sa présence. À Pompéi, il fera la rencontre de celle qu'il croit être le fantôme de Gradiva, avec qui il entretiendra de longues conversations, avant de découvrir, après un grand chemin, qu'il s'agit en fait d'une jeune fille (Zoé) qu'il connaissait jadis, et que son délire l'avait empêché de reconnaître. On lira sur ce point la lecture que propose Jacques Derrida du texte de Jensen à partir de l'essai de Freud (Derrida 1995 : 149-155).

2. On retrouve une reproduction de Gradiva dans le catalogue de I'exposition Voyage to Italy (2006).

En ligne: http://malcontenta.blog.lemonde.fr/files/408pxgradivap1030638.jpg (page consultée le 10 juin 2007).

\section{RÉFÉREN CES BIBLIO G RAPH IQ U ES}

BuRGIN, V. [1996] : In/Different Spaces, Berkeley, University of California Press;

[2004] : The Remembered Film, Londres, Reaktion Books;

Cantz, 79-87.

[2006] : "The Shadow and the Ruin », dans H. von Amelunxen (dir.), Voyage to Italy, catalogue de l'exposition, Ostfildern, Hatje

DERRIDA, J. [1995] : Mal d'Archive. Une impression freudienne, Paris, Éd. Galilée, coll. «Incises ».

Dubols, P. [1981] : «Figures de Ruine: notes pour une esthétique de l'index», Rivista di Estetica, vol. XXI, n 8, 8-20.

FREUD, S. [(1907) 1971] : Délire et rêves dans la "Gradiva» de Jensen, Paris, Gallimard.

SIMMEL, G. [(1907) 1998] : "Les ruines. Un essai d'esthétique», La Parure et autres essais, Paris, Éd. de la maison des sciences de l'homme, 116-117. 
Basilica I, 2006, 12 × $8 \mathrm{~cm}$ (chaque image).
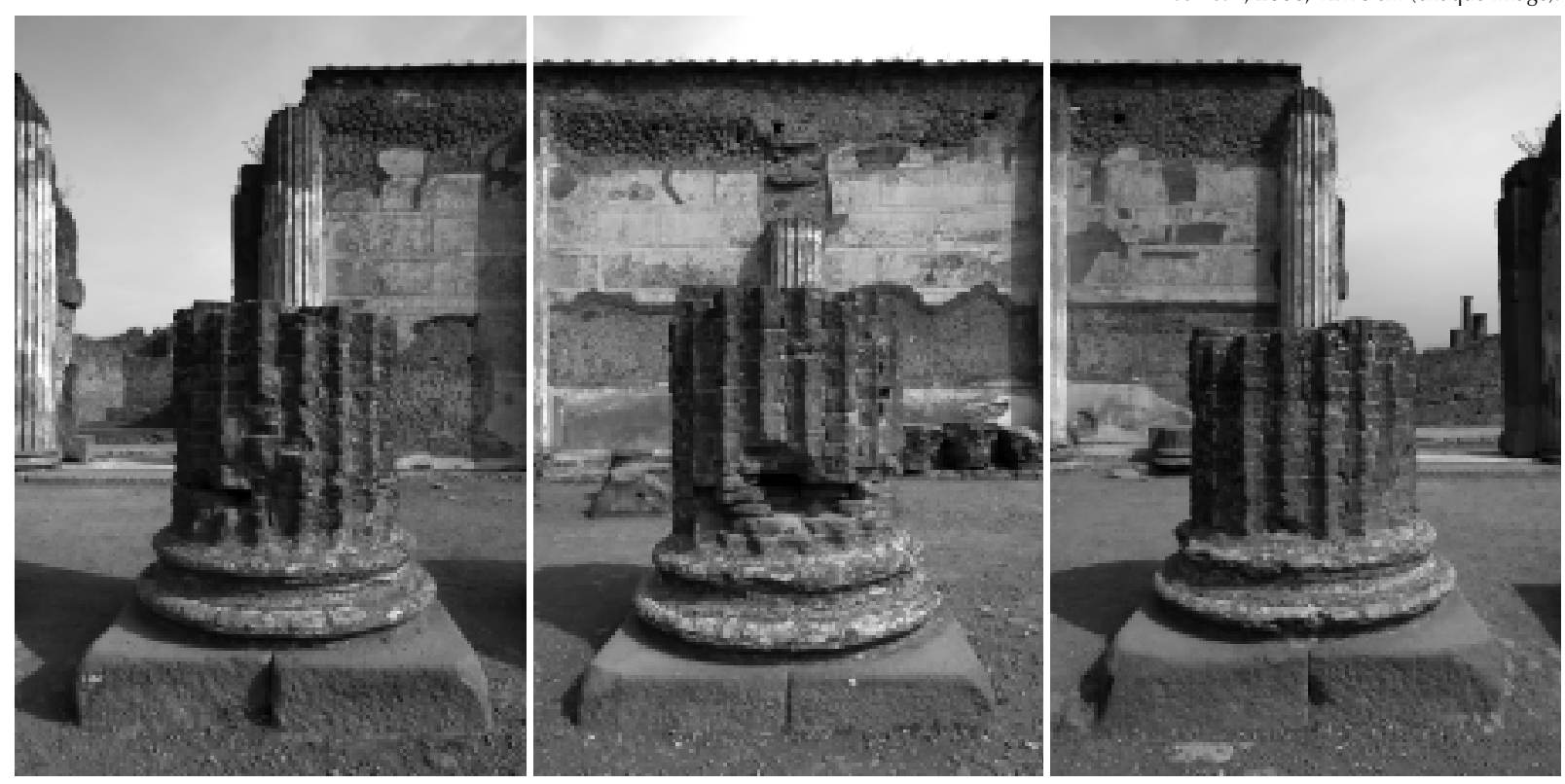


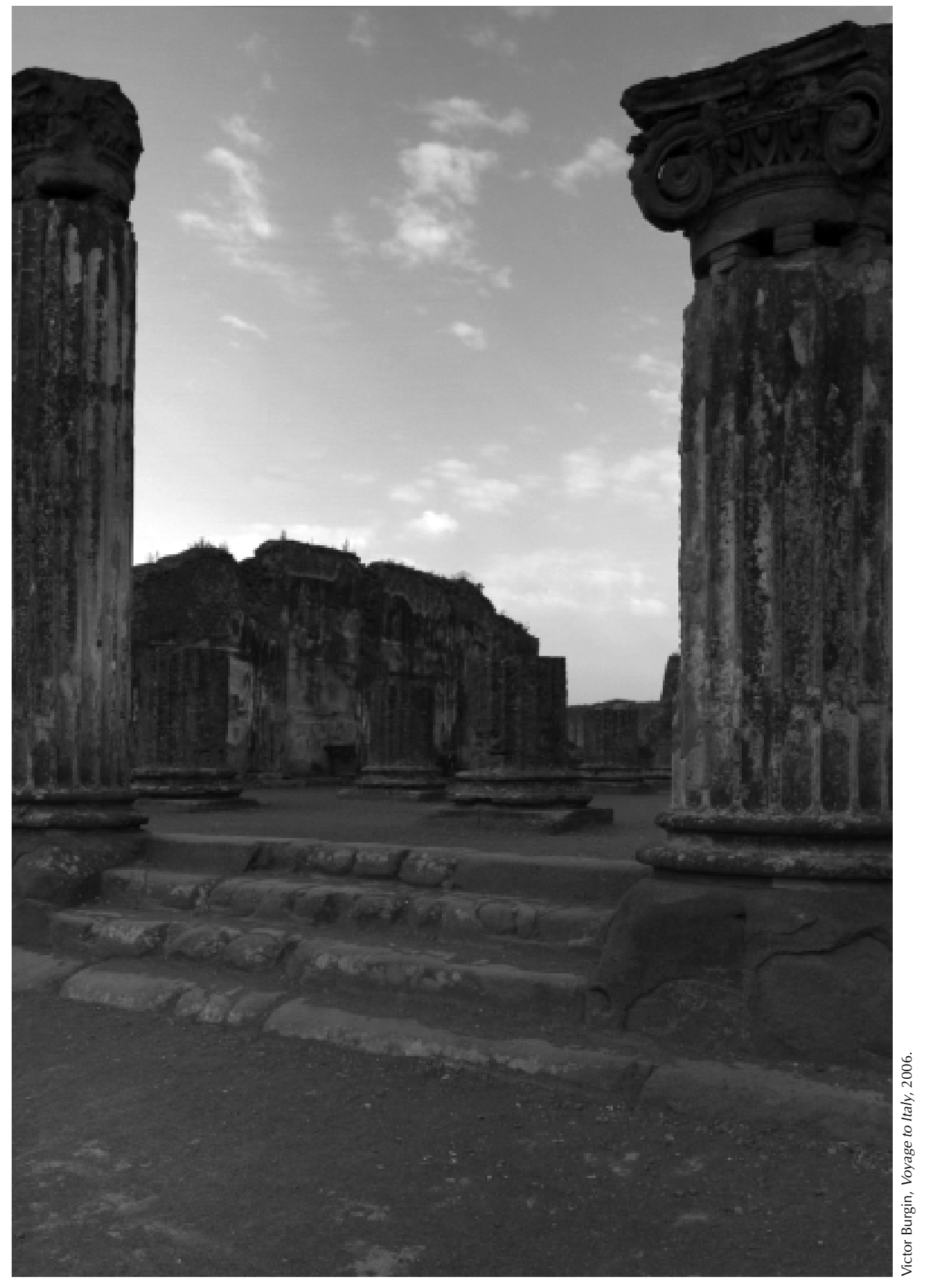




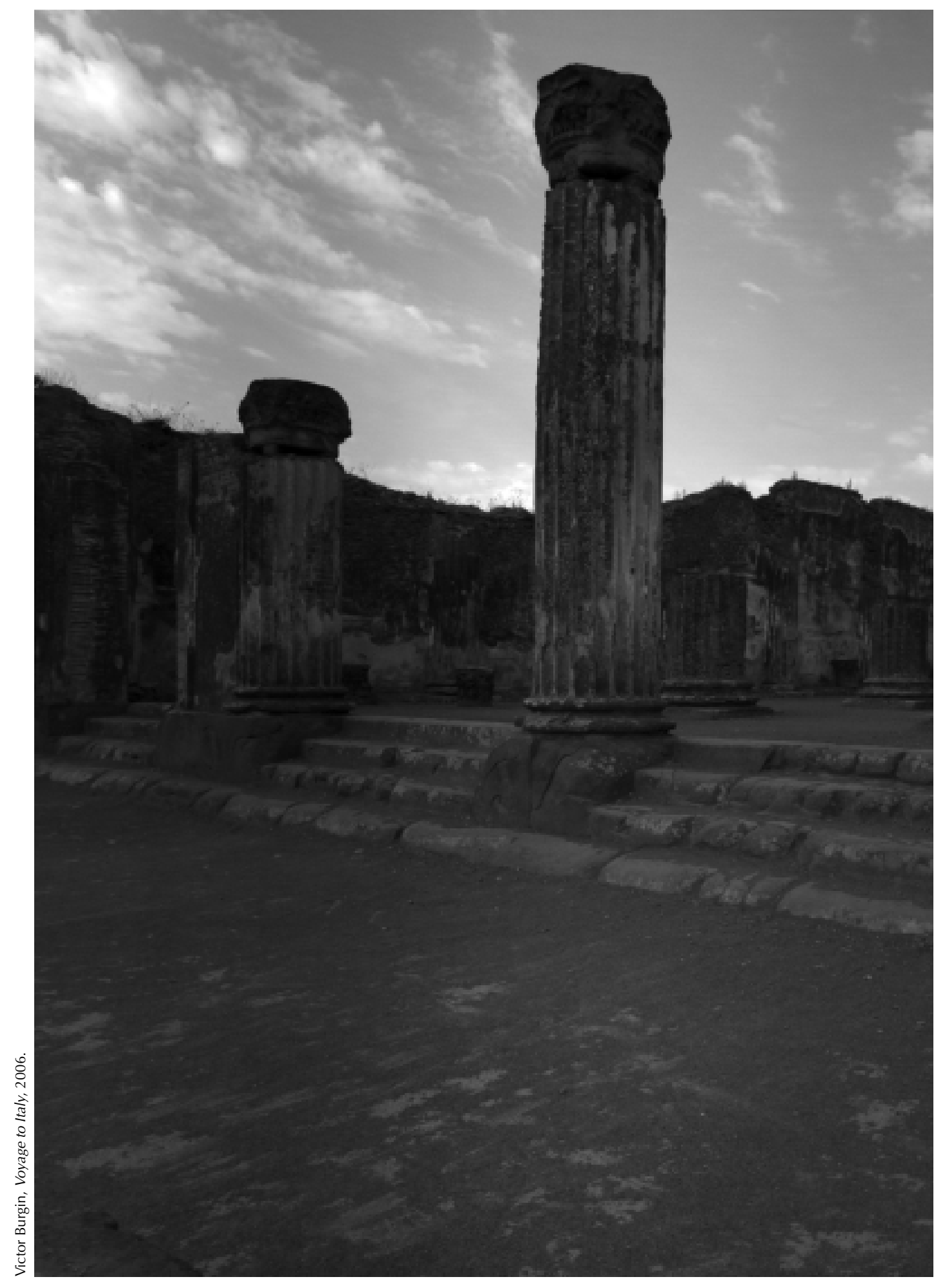




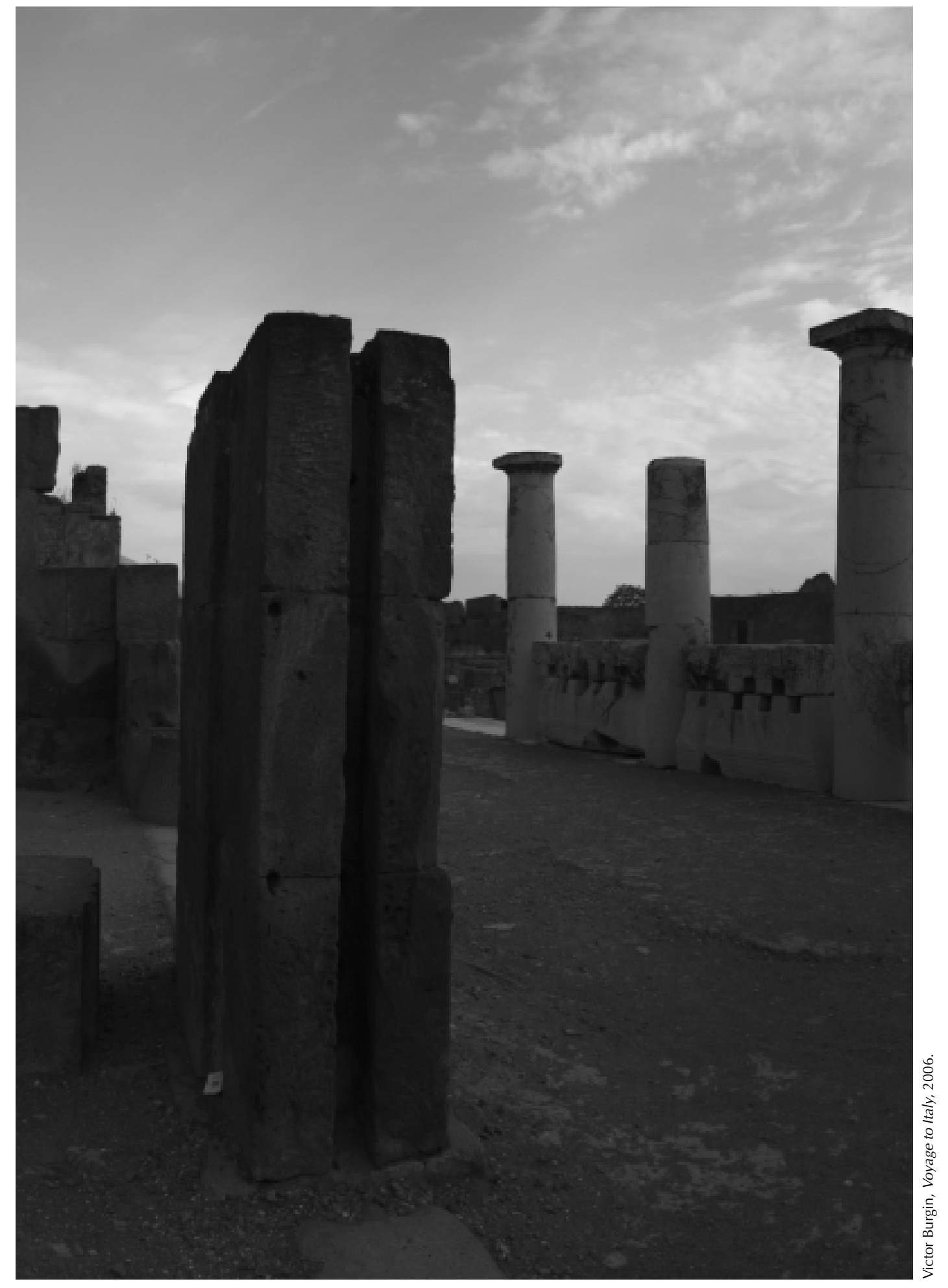




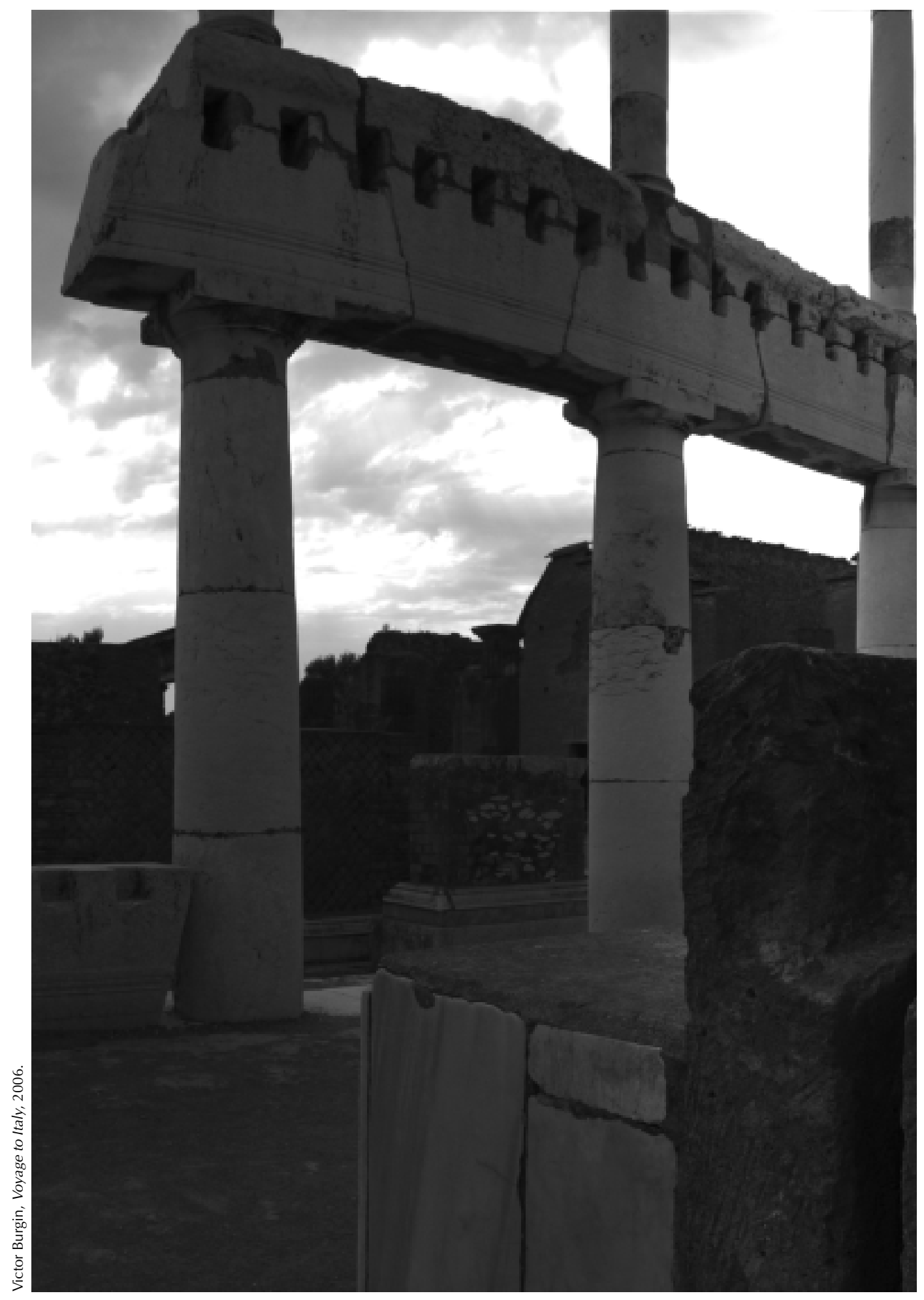




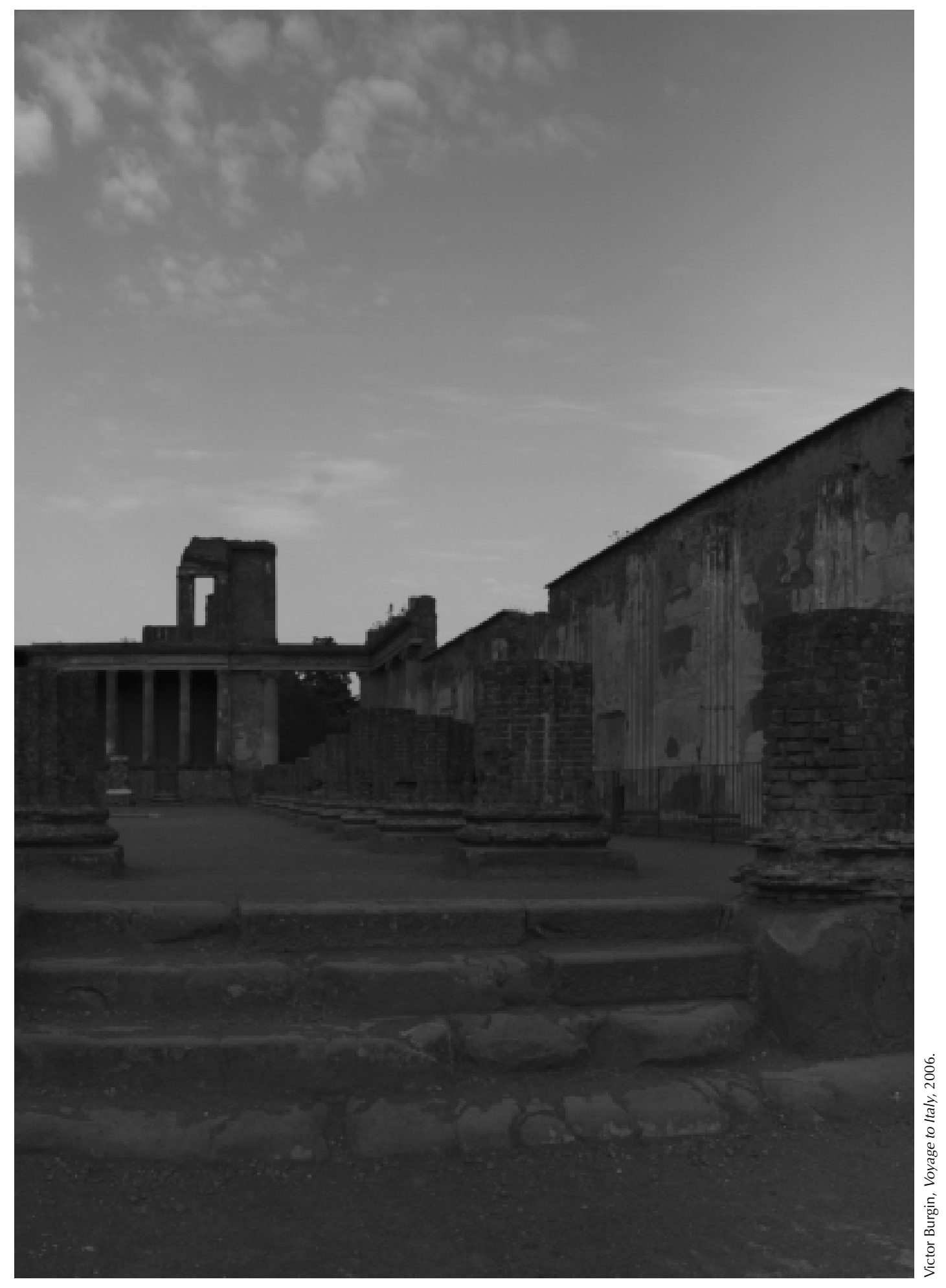

